\title{
USO E PERCEPÇÕES DA ALIMENTAÇÃO ALTERNATIVA NO ESTADO DA BAHIA: UM ESTUDO PRELIMINAR
}

\author{
USE AND PERCEPTIONS OF ALTERNATIVE FOOD IN \\ THE STATE OF BAHIA: A PRELIMINARY STUDY
}

\author{
Lígia Amparo da Silva SANTOS' \\ Aline Maria Peixoto LIMA² \\ Ivana Vieira PASSOS \\ Leonor Maria Pacheco SANTOS ${ }^{3}$ \\ Micheli Dantas SOARES ${ }^{4}$ \\ Sandra Maria Chaves dos SANTOS
}

RESUMO

\begin{abstract}
O presente estudo faz parte de um projeto maior, intitulado "Avaliação das Políticas Públicas na Área de Alimentação e Nutrição Implementadas no Estado da Bahia, no período de 1994 a 1997". Teve como objetivo reconhecer como vem se dando o uso da alimentação alternativa nas populações de baixa renda no Estado da Bahia, bem como identificar a percepção que as mesmas têm sobre esta prática. Foram aplicados questionários em 1380 domicílios distribuídos em localidades urbanas e rurais de 36 municípios. O estudo revelou que 57,2\% da população estudada já ouviu falar da alimentação alternativa e, deste percentual, 46,8\% fazem uso desta prática. O principal veiculador do uso da alimentação alternativa, conforme esperado, foi a Pastoral da Criança, referida por 33,6\% dos entrevistados. Do total dos entrevistados, 70,7\% demonstraram uma percepção positiva sobre a alimentação alternativa como uma estratégia que contribui para a melhoria das condições de saúde.
\end{abstract}

Termos de indexação: alimentação alternativa, nutrição, hábitos alimentares; métodos de alimentação.

\begin{abstract}
The present study is part of a major project, entitled "Assessment of Public Policy in Food and Nutrition Implemented in the state of Bahia, between 1994-1997". The objective was to recognise how alternative food is being used in population with low income in Bahia as well as to identify their perceptions of these practices. Questionnaires were applied in 1.380 domiciles in urban and rural areas spread over 36 cities. The study revealed that $57.2 \%$ of the population has already heard about alternative food and, among these, $46.8 \%$ have used this food strategy. The main disseminator of the use of alternative food was, as expected, the "Pastoral da Criança"(Social Organization of the Catholic Church for Children) referred by $33.6 \%$ of the interviewees. This study also observed that $70.7 \%$ of the interviewees demonstrated a positive perception about alternative food as a strategy that contributes to improve the health conditions.

Index terms: alternative food, nutrition, food habits, feeding methods.

\footnotetext{
(1) Departamento de Ciências da Nutrição, Escola de Nutrição, Universidade Federal da Bahia. Rua Araújo Pinho, 36, Canelas, 40110-170, Salvador, BA, Brasil. Correspondência para/Correspondence to: L.A.S.SANTOS.E-mail: amparo@ufba.br

(2) Programa de Pós-Graduação em Nutrição, Escola de Nutrição, Universidade Federal da Bahia

(3) Departamento de Nutrição, Universidade Federal de Pernambuco.

(4) Programa de Pós-Graduação em Saúde Coletiva, Universidade Estadual de Feira de Santana.
} 


\section{INTRODUÇÃO}

A alimentação alternativa tem sido uma prática bastante disseminada no Brasil a partir do início da década de 80 , principalmente para grupos considerados biossocialmente vulneráveis, como crianças e gestantes. Consiste, então, entre seus defensores e disseminadores, em uma estratégia de combate à fome e desnutrição colocada especialmente para as classes populares cujo acesso aos alimentos tem sido historicamente negado.

A alimentação alternativa tem sido definida como "a proposta de promover na dieta brasileira o uso de alimentos tradicionais e não tradicionais, ricos em vitaminas e minerais, que são acessíveis a toda população". Entre os alimentos que são promovidos encontram-se: farelos (especialmente os de trigo e arroz), folhas verdes (de beterraba, taioba, caruru, bredo, batata-doce, cenoura), pó de folhas (que formam a chamada multimistura), cascas (de verduras e frutas como banana, abóbora e ainda casca de ovo) e sementes (gergelim, melancia, abóbora) (Debessautet, 1992).

Um dos pontos chaves da estratégia da alimentação alternativa é a multimistura, que consiste na mistura de pós de farelos, folhas, cascas e sementes de diversos subprodutos a serem acrescentados à dieta, visando o aumento de seu valor nutricional. O princípio da multimistura, segundo Brandão, citado por Mascarenhas (1994), é que a qualidade é dada pela variedade. Trata-se também, possivelmente, da proposta mais questionada, seja quanto ao valor nutricional intrínseco da preparação, seja quanto à sua adequação frente às necessidades nutricionais dos grupos etários e fisiológicos aos quais prioritariamente pretende contemplar.

Segundo Bittencourt (1998), a promoção do uso da alimentação alternativa foi desenvolvida pelos médicos Clara e Rubens Brandão há 25 anos. Contudo, a sua eficácia em relação à desnutrição tem sido discutida em todo o país. A Pastoral da Criança da Confederação Nacional de Bispos do Brasil (CNBB) tem a alimentação alternativa como uma das linhas de suas ações básicas de saúde, nutrição e educação. A participação comunitária através dos líderes da Pastoral constitui a base sólida para a disseminação desta prática.

A estratégia da alimentação alternativa tem feito parte das intervenções públicas na área de Alimentação e Nutrição em alguns estados e municípios brasileiros. Debessautet (1992), citado por Bittencourt (1998), refere a difusão desta prática há nove anos por alguns serviços da rede pública de saúde e educação. Em Salvador, nos meados da década de oitenta, creches conveniadas com a Secretaria Estadual de Saúde da Bahia adotaram a alimentação alternativa (Santos, 1996a). A nível central, também parece haver um apoio do Ministério da Saúde a esta prática.
No Estado da Bahia, a Pastoral da Criança atua em 342 municípios, com 14510 líderes comunitários, cobrindo 4153 comunidades e abrangendo uma média mensal de 116954 famílias e 180289 crianças (Pastoral da Criança, 2000). A dimensão do trabalho da Pastoral, principal disseminadora da prática da alimentação alternativa no Estado, indica a necessidade de maior reflexão sobre esta estratégia.

Conforme anteriormente referido, esta prática tem gerado polêmica. De um lado, temos a Pastoral da Criança, que afirma ter obtido resultados bastante satisfatórios, em suas comunidades de atuação, na recuperação especialmente de crianças com déficit nutricional, e de outro, a comunidade científica da área de alimentação e nutrição, que levanta inúmeros questionamentos a esta prática.

Uma série de eventos foram realizados, sobretudo nos anos de 1995 e 1996, período este caracterizado como de grande efervescência, quando as discussões giraram em torno da insuficiência de investigações científicas quanto aos seguintes aspectos: eficácia nutricional, biodisponibilidade dos nutrientes em função do alto valor de fatores antinutricionais, qualidade microbiológica e físico-química dos subprodutos utilizados (Quadro 1). Somado a isto, Bittencourt (1998) analisando seis estudos epidemiológicos, concluiu que há muitas ambigüidades, lacunas e contradições no que tange aos aspectos metodológicos, levantando dúvida quanto à confiabilidade dos resultados encontrados.

Analisando tal debate, percebe-se que o enfoque central sobre a alimentação alternativa é a sua dimensão técnico-científica, deixando marginalizada a discussão sobre o seu conteúdo sócio-político e ideológico. Neste contexto, considera-se a existência de lacunas em termos de estudos que discutam qual poderia ser o papel da alimentação alternativa no bojo das políticas públicas de alimentação e nutrição, tendo como referencial a construção da Segurança Alimentar.

Recentemente, a Pastoral da Criança vem revisitando esta questão, modificando o termo alimentação alternativa por "alimentação enriquecida" e propondo ampliação de enfoque com a inclusão de aspectos do conceito de Segurança Alimentar (Pastoral da Criança, 1999).

Um outro aspecto importante diz respeito à representação social da prática da alimentação alternativa. Enquanto discute-se dentro da comunidade científica sua validade ou não, presume-se que uma parcela significativa da população brasileira, particularmente das classes populares, esteja promovendo ou utilizando a alimentação alternativa em sua dieta cotidiana. Para muitos, trata-se de um resgate da alimentação popular, compreendendo-a como solução para os problemas de saúde e nutrição, quando "o melhor do alimento é jogado fora" (Santos, 1996b). 
Quadro 1. Síntese das principais conclusões e recomendações de três eventos científicos.

\begin{tabular}{|c|c|c|c|}
\hline & $\begin{array}{l}\text { Reunião Interinstitucional sobre opções para } \\
\text { melhoria alimentar e nutricional da população } \\
\text { (1995). }\end{array}$ & $\begin{array}{l}\text { Conclusões do workshop sobre Recuperação } \\
\text { nutricional de grupos populacionais de baixa } \\
\text { renda, Análise crítica (1996). }\end{array}$ & Posicionamento do CFN quanto a multimistura \\
\hline Participantes & $\begin{array}{l}\text { Ministério da Saúde, Coordenação Nacional da Pas- } \\
\text { toral da Criança, Sociedade Brasileira de Pediatria, } \\
\text { Universidadede SãoPaulo, Fundação Osvaldo Cruz, } \\
\text { Coordenação Materno Infantil, Empresa Brasileira } \\
\text { dePesquisa Agropecuária, Fundo das Nações Unidas } \\
\text { para Infância, Instituto Nacional de Alimentação e } \\
\text { Nutrição, Instituto Materno Infantil de Pernambuco, } \\
\text { Universidade Federal de Pelotas, Universidade } \\
\text { Federal do Rio Grande do Sul. }\end{array}$ & $\begin{array}{l}\text { Documento resultante do evento organizado } \\
\text { pela Sociedade Brasileira de Alimentação e } \\
\text { Nutrição; Sociedade Brasileira de Alimentos, } \\
\text { Sociedade Brasileira de Pediatria. }\end{array}$ & $\begin{array}{l}\text { O CFN respaldou-se no informe técnico } \\
\text { "ProgramasEmergenciaisdeCombateàFomee } \\
\text { o Uso de Subprodutos de Alimentos" da } \\
\text { Unicamp. }\end{array}$ \\
\hline $\begin{array}{l}\text { Conclusõese } \\
\text { Recomendações }\end{array}$ & $\begin{array}{l}\text { Hánecessidade de um maior esclarecimento quanto } \\
\text { a aspectos da biodisponibilidade de nutrientes e } \\
\text { eventuais riscosdecontaminação. Propõe duas linhas } \\
\text { prioritárias de pesquisa:-identificaçãoenormatização } \\
\text { de procedimentos para o preparo da multimistura } \\
\text { nocontexto das ações básicas de saúde; - nutriçãoe } \\
\text { educação da Pastoral da Criança. Outros quatro } \\
\text { tópicos abordam: a necessidade de apoio e } \\
\text { fortalecimento das ações em nível comunitário; as } \\
\text { interações institucionais; o controle de qualidadea } \\
\text { nível central, comunitário e familiar; e o papel da } \\
\text { comunicação sobre os trabalhos neste campo. }\end{array}$ & $\begin{array}{l}\text { - O evento questionou o apoio expresso pelo } \\
\text { Ministério da Saúde ao uso de alimentos ditos } \\
\text { alternativos em populações carentes, apesar } \\
\text { das contestações científicas. Colocou ainda a } \\
\text { necessidade de haver registros na divisão de } \\
\text { alimentos do Departamento Técnico } \\
\text { Normativo daSecretaria deVigilância Sanitária } \\
\text { (MS), bem como de desenvolver estudos que } \\
\text { avaliema eficácia nutricional do farelode arroz. } \\
\text { Tecem críticas ainda a metodologia dos } \\
\text { trabalhos realizados. }\end{array}$ & $\begin{array}{l}\text {-Consideraa "urgentenecessidade de definição } \\
\text { de uma Política Nacional de Alimentação e } \\
\text { Nutrição como prioridade de Governo, que } \\
\text { resgate a cidadania e o direito ao alimento, à } \\
\text { saúde eà vida." }\end{array}$ \\
\hline
\end{tabular}

Por outro lado, há os que consideram tratar-se de restos ou sobras de alimentos. Tais colocações apontam para a existência de um debate dentro das classes populares travado entre os que acreditam na multimistura, promovem-na e/ou utilizam-na e aqueles que rejeitam a sua utilização resistem a ela. Esta condição traz dúvida sobre o nível de adesão no meio familiar a esta prática.

A interpretação dos dilemas das classes populares sobre alimentação alternativa é, todavia, uma outra lacuna nas investigações acadêmicas, quer seja enquanto significação em si de uma alimentação alternativa que não pode ser considerada como tal, uma vez que não se trata necessariamente de uma escolha, quer seja enquanto representação de uma alimentação que impõe novos hábitos, novos conceitos simbólicos. Neste sentido, as idéias sobre o alimento, em especial sobre a alimentação alternativa, parecem imbuídas de significados que, de algum modo, representam o cuidado com o corpo e suas necessidades.

Este estudo pretende inicialmente identificar como vem se dando o uso da alimentação alternativa nas populações de baixa renda no Estado da Bahia, bem como conhecer a percepção que as mesmas têm sobre esta prática. Trata-se de uma etapa inicial sobre a investigação deste tema.

\section{CASUÍSTICA E MÉTODOS}

Este trabalho é parte de um projeto maior, intitulado “Avaliação das Políticas Públicas na Área de Alimentação e Nutrição Implementadas no Estado da Bahia, no período de 1994 a 1997". Embora as práticas de alimentação alternativa não se constituam de fato em uma política pública, a sua inclusão como um dos subprojetos estudados deveu-se a sua relevância no campo da alimentação e nutrição.

Os 36 municípios estudados no interior do Estado da Bahia foram selecionados tendo como critério pertencer ao Programa Comunidade Solidária do Governo Federal. O estudo empírico contemplou o levantamento domiciliar de informações sobre a Alimentação Alternativa, bem como sobre a existência e conhecimento desta estratégia pelo conjunto de potenciais beneficiários. Nesta perspectiva, em cada um dos municípios estudados, realizou-se o levantamento em 40 domicílios, sendo 20 no extrato urbano e 20 no extrato rural.

A escolha dos bairros, ruas ou povoados foi não probabilística e do tipo intencional. Em outras palavras, considerando tratar-se de parte de uma avaliação de políticas públicas para população de baixa renda, o estudo teve a intenção explícita de identificar as áreas mais pobres dos municípios para a realização do levantamento domiciliar. Estas localidades foram selecionadas através da triangulação de informação, pela aplicação parcial da metodologia da estimativa rápida. Neste caso, buscou-se identificar em cada município um conjunto de informantes-chaves que, através da inserção em setores da prefeitura ou pelo desenvolvimento de trabalhos sociais no município, possuíssem informações sobre as áreas mais pobres na zona urbana e rural. No geral, estes informantes foram o próprio prefeito, a primeira-dama, agentes comunitários de saúde, 
padres e freiras da Pastoral da Criança e lideranças do sindicato de trabalhadores rurais.

Em uma etapa inicial, a três destes colaboradores questionou-se qual era a área mais pobre do município no perímetro urbano e na zona rural. Quando as três respostas coincidiam, a área era selecionada para o estudo. Não havendo coincidência a partir do questionamento aos três primeiros entrevistados, iniciava-se outra etapa de questões a novos colaboradores. E assim foi feito sucessivamente até serem obtidas três respostas coincidentes.

Selecionado o domicílio, o entrevistado deveria ser preferencialmente o chefe da casa ou o responsável. O instrumento utilizado na coleta de dados foi um questionário composto de questões abertas e fechadas. Na caracterização social das famílias, utilizou-se a classificação adaptada de Lopes (1993) sobre necessidades básicas satisfeitas e insatisfeitas. Este indicador baseia-se na combinação de certas características físicas de habitação que apontam para más condições sanitárias, com um índice de alta densidade por dormitório, bem como com outro índice para caracterizar condições sociais desfavoráveis. O autor utiliza este indicador, cruzando-o com a linha de pobreza para uma análise mais acurada da mesma.

No presente estudo utilizamos apenas o indicador de necessidades básicas satisfeitas e insatisfeitas, usando os seguintes índices: tipo de domicílio; abastecimento de água, esgoto sanitário e presença de vaso sanitário, através da aplicação de um questionário estruturado de questões fechadas que contemplou o levantamento dos referidos índices.

Desta forma, foram aplicados 1440 questionários em domicílios. Porém, devido a uma perda amostral de 4,16\%, apresentamos os dados de 1380 deles. Os dados produzidos foram analisados utilizando os Softwares Access e SPSS. O tratamento dos dados foi obtido através de estatística simples para destacar informações e permitir interpretações, considerando o objeto do estudo.

\section{RESULTADOS E DISCUSSÃO}

Das 1380 famílias entrevistadas, a partir dos índices adaptados de Lopes (1993), 95,2\% destas têm suas necessidades básicas insatisfeitas, evidenciando a precariedade socioeconômica dos domicílios estudados e caracterizando os entrevistados como população-alvo das estratégias de incentivo ao uso da alimentação alternativa.

Em relação ao conhecimento e uso da alimentação alternativa, os resultados demonstraram ser expressivos: $57,2 \%$ dos entrevistados já tinham ouvido falar da alimentação alternativa e, deste universo, 46,8\% referiram fazer uso. Do percentual de 53,2\% que referiu não fazer uso da alimentação alternativa, os principais motivos foram a falta de interesse $(34,0 \%)$, a falta de orientação $(23,3 \%)$, a falta de credibilidade na estratégia $(13,6 \%)$ e outros motivos $(29,1 \%)$ (Tabela 1$)$.

Tabela 1. Razões para a não utilização da alimentação alternativa em populações urbana e rural de 36 municípios do Estado da Bahia, 1997 a 1998.

\begin{tabular}{lcc}
\hline \multirow{2}{*}{ Razões } & \multicolumn{2}{c}{ Freqüência } \\
\cline { 2 - 3 } & $\mathrm{n}$ & $\%$ \\
\hline Há falta de interesse & 143 & 34,0 \\
Há falta de orientação & 98 & 23,3 \\
Há falta de credibilidade & 57 & 13,6 \\
Não é população-alvo & 24 & 5,7 \\
É trabalhoso para fazer & 22 & 5,2 \\
Não precisa & 12 & 2,7 \\
Tem receio de utilizar & 12 & 2,7 \\
Outros & 54 & 12,8 \\
\hline Total & 420 & 100,0 \\
\hline
\end{tabular}

Fonte: Pesquisa de Campo.

Os dados da não utilização da alimentação alternativa possibilitam duas direções de interpretação: uma que pode revelar uma lacuna na estratégia de divulgação da proposta, a exemplo da falta de orientação; e outra que revela a possível "rejeição" ao consumo, exemplificada pela falta de interesse.

Ao investigar a opinião da população estudada sobre a alimentação alternativa, observou-se que 70,7\% têm uma percepção positiva da mesma. Os relatos apresentaram dois enfoques: um referindo-se à melhoria da saúde $(87,5 \%)$ e outro, relacionando alimentação alternativa enquanto estratégia alimentar (12,5\%), quer seja por necessidade, complementação ou aproveitamento integral dos alimentos (Tabela 2). Esta percepção possivelmente é resultado do trabalho que a Pastoral da Criança desenvolve, não associado à falta de opção "para quem não tem o que comer" mas sim disseminado como uma prática que contribui para a melhoria do estado de saúde, como vem sendo colocado nos mais recentes documentos onde há a incorporação do conceito de Segurança Alimentar e Nutricional.

Em relação à percepção negativa referida pelos entrevistados, observa-se que a rejeição desta prática está relacionada à não credibilidade, à baixa resolubilidade e às características sensoriais dos produtos utilizados.

Por outro lado, os entrevistados que têm uma percepção negativa podem estar referendando a prática da alimentação alternativa como uma prática direcionada para deficiências alimentares e nutricionais em grupos populacionais específicos, não sendo identificada portanto, como uma prática universal 
voltada para o aproveitamento integral dos alimentos. Embora não muito expressivo, um percentual de 5,7\% (Tabela 2) dos entrevistados referiu não adesão à prática pelo fato de não pertencer à população-alvo, e na categoria outros foi citado como motivo "não fazer a catequese", demostrando uma identificação entre a alimentação alternativa e a religião católica.

Na tentativa de identificar quem preferencialmente consome estes alimentos, observou-se que as crianças são os usuários potenciais (40,9\%), seguidas por toda a família $(35,2 \%)$ e pelas mães e crianças $(5,7 \%)$ (Figura 1). Estes dados revelam que, apesar de ser uma prática que por longo tempo foi dirigida para grupos biologicamente vulneráveis, a utilização da alimentação alternativa parece estar sendo incorporada por toda a família, tendo em vista que apenas $3,8 \%$ das famílias apontaram as crianças desnutridas como usuárias potenciais deste tipo de alimentação.

As opiniões dos entrevistados sobre a alimentação alternativa corroboram com estes dados, uma vez que estão mais relacionadas com a melhoria da saúde em geral, como pôde ser observado nos relatos: "... fortalece o organismo, sangue e ossos....", ".... ajuda a engordar e crescer ...", "... serve para fraqueza, diarréia e desnutrição". E ainda verificou-se que apenas $7,9 \%$ da população entrevistada demonstrou uma percepção positiva por conta da recuperação do estado nutricional (Tabela 2).

Quanto às fontes de informação sobre a estratégia, $33,6 \%$ haviam obtido alguma informação sobre alimentação alternativa através da Pastoral da Criança. Parentes e vizinhos têm uma participação importante como agentes multiplicadores na difusão desta prática, tendo sido citados em 22,4\% dos domicílios. Os meios de comunicação como veículo de acesso à informação sobre tais práticas foram referidos em 10,3\% dos casos (Figura 2). Vale ressaltar que a participação de parentes e vizinhos na divulgação pode estar indicando o reflexo da ação da Pastoral, posto que a inserção desta é sobretudo comunitária.

\section{CONCLUSÃO}

No conjunto das intervenções sociais em alimentação e nutrição não há tradição de estudos sobre opiniões, atitudes e expectativas dos destinatários. As intervenções são desenhadas a partir de um ponto de vista unilateral, descartando esta perspectiva. No entanto, a percepção dos usuários é de fundamental importância tanto para o sucesso da estratégia a ser implementada, como para sua avaliação.

Tabela 2. Opinião dos entrevistados sobre a alimentação alternativa em populações urbana e rural de 36 municípios do Estado da Bahia, 1997 a 1998.

\begin{tabular}{|c|c|c|c|}
\hline \multirow{2}{*}{ Percepção } & \multirow{2}{*}{ Opinião } & \multicolumn{2}{|c|}{ Freqüência } \\
\hline & & $\mathrm{n}$ & $\%$ \\
\hline \multirow[t]{9}{*}{ Positiva } & Faz bem para a saúde & 211 & 26,7 \\
\hline & É fonte de vitamina & 91 & 11,5 \\
\hline & Fortalece o organismo & 74 & 9,4 \\
\hline & Recupera a desnutrição & 62 & 7,9 \\
\hline & Complementa a alimentação & 42 & 5,4 \\
\hline & Evita doenças & 30 & 3,8 \\
\hline & Serve como remédio & 20 & 2,5 \\
\hline & Aproveita os alimentos & 16 & 2,0 \\
\hline & Ajuda quem necessita & 12 & 1,5 \\
\hline Subtotal & & 558 & 70,7 \\
\hline \multirow[t]{3}{*}{ Negativa } & Éruim & 19 & 2,4 \\
\hline & Testou e não obteve resultados & 15 & 1,9 \\
\hline & Não tem fé & 38 & 4,8 \\
\hline Subtotal & & 72 & 9,1 \\
\hline Sem opinião formada & & 65 & 8,2 \\
\hline Indiferente & & 18 & 2,4 \\
\hline Sem informação & & 159 & 20,2 \\
\hline Total & & 789 & 100,0 \\
\hline
\end{tabular}

Fonte: Pesquisa de Campo. 


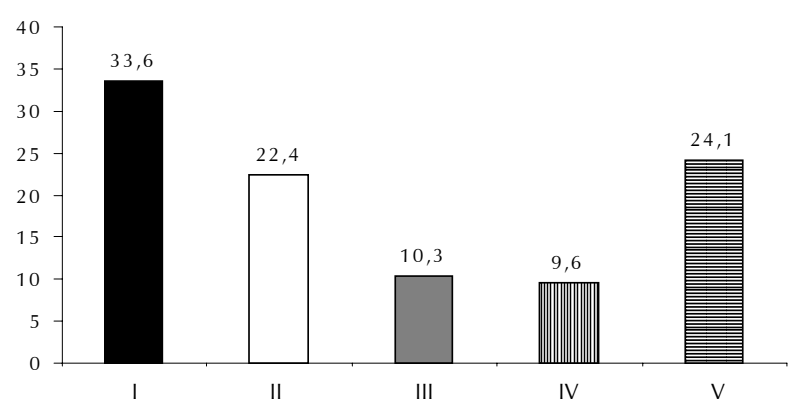

Figura 1. Fontes de informação sobre alimentação alternativa em populações urbana e rural de 36 municípios do Estado da Bahia, 1997 a 1998

Nota: $I=$ Pastoral da criança; $I I=$ Comunidade; $I I I=$ Meios de comunicação; $\mathrm{IV}=$ Escola $; \mathrm{V}=$ Outros

O estudo revelou que foi relativamente expressiva não só a informação sobre a alimentação alternativa, como também a sua utilização pela população. Ressalta-se, entretanto, que o estudo trabalhou com a auto-referência da utilização dos subprodutos, o que não revela a real participação da alimentação alternativa na dieta cotidiana dos usuários, conforme os dados encontrados na avaliação de freqüência e quantidade de consumo alimentar.

O principal veiculador da prática da alimentação alternativa, conforme esperado, é a Pastoral da Criança, no entanto, observou-se uma pequena incorporação desta dentro dos espaços institucionais tanto de educação quanto de promoção à saúde.

Por fim, a população estudada revelou uma percepção "positiva" em relação à alimentação alternativa enquanto uma estratégia de melhoria das condições de saúde, bem como de aproveitamento dos recursos alimentares. Sendo a Pastoral o principal veiculador desta prática, a percepção que emerge da comunidade, via de regra, reflete o próprio discurso da Pastoral. Desta forma, este fator pode dificultar o debate sobre o tema no seio das classes populares, uma vez que o seu principal disseminador tem a sua prática permeada por uma dimensão religiosa. Neste sentido, sugerimos maiores investigações que contribuam para elucidar tais questões.

\section{REFERÊNCIAS BIBLIOGRÁFICAS}

BITTENCOURT, S.A. Uma alternativa para política nutricional brasileira? Cadernos de Saúde Pública, Rio de Janeiro, v.14, n.13, p.629-636, 1998.

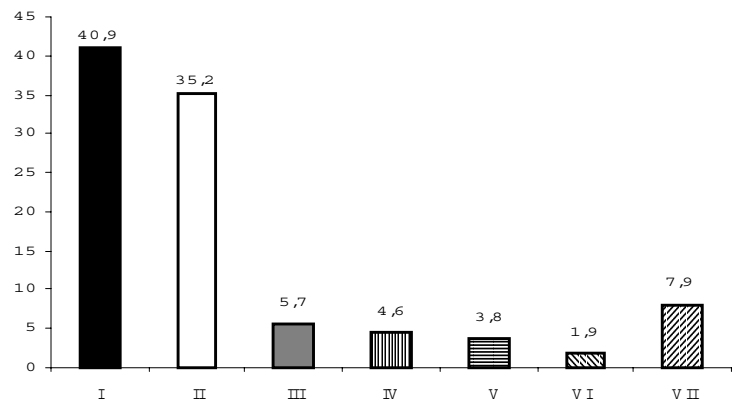

Figura 2. Grupo populacional que consome a alimentação alternativa em populações urbana e rural de 36 municípios do Estado da Bahia, 1997 a 1998.

Nota: $I=$ Crianças; $I I=$ Toda a Família; $I I I=$ Mães e Crianças; IV= Adultos; $\mathrm{V}=$ Crianças de risco; $\mathrm{VI}=$ Outros $; \mathrm{VII}=$ Sem Informação

DEBESSAUTET, I. Estudio de las bases cientificas para el uso de alimentos alternativos en la nutrición humana. Brasília : INAN, 1992. 92p. (Mimeografado).

LOPES, R.B.L. Brasil, 1989: um estudo sócio-econômico da indigência e da pobreza urbanas. Caderno de Pesquisa, Campinas, n.25, 1993. 60p.

MASCARENHAS, M.G. et al. Soluções ao alcance das mãos. Globo Ciência, n.51, p.12-16, 1994.

PASTORAL DA CRIANÇA. Ações básicas. Brasil, 1999. Disponível em: <URL:http:// www.rebidia.org.br/pastoral/ fr_acoes.html>. Acesso em: 12 dez. 2000.

PASTORAL DA CRIANÇA. Situação de Abrangência da Pastoral da Criança: $2^{\circ}$ Trimestre de 2000. Disponível em: < URL: http://www.rebidia.org/estados/UF_BA.HTM>. Acesso em: 12 dez. 2000.

SANTOS, L.A.S. Saúde e nutrição no município de Valente, BA. Valente, Bahia, 1996a. 8p. (Relatório Técnico apresentado a APAEB).

SANTOS, L.A.S. Representações e práticas da alimentação alternativa. Proposta de trabalho para dissertação de mestrado em saúde pública apresentada à Escola Nacional de Saúde Pública, Rio de Janeiro, 1996b 13 p. (Mimeografado).

Recebido para publicação em 6 de julho de 2000 e aecito em 2 de agosto de 2001. 